\title{
Percutaneous Image-Guided Cryoablation for the Treatment of Phantom Limb Pain in Amputees: A Pilot Study
}

\author{
J. David Prologo, MD, Charles A. Gilliland, MD, Michael Miller, MD, Paul Harkey, MD, Jackie \\ Knight, NP-C, Darren Kies, MD, C. Matthew Hawkins, MD, David Corn, MS, David K. \\ Monson, MD, Faramarz Edalat, MD, Sean Dariushnia, MD, and Luke Brewster, MD \\ Division of Interventional Radiology (J.D.P., C.A.G., M.M., D.K., C.M.H., S.D.), Department of \\ Radiology and Imaging Sciences (P.H., F.E.), Department of Orthopaedic Surgery (D.K.M.), and \\ Department of Surgery (L.B.), Emory University School of Medicine, 1364 Clifton Road, NE Suite \\ D112, Atlanta, GA 30322; and Emory Clinic (J.K., D.C.), Atlanta, Georgia
}

\section{Abstract \\ Purpose-To prospectively evaluate percutaneous image-guided nerve cryoablation for treatment of refractory phantom limb pain (PLP) in a pilot cohort for purposes of deriving parameters to design a larger, randomized, parallel-armed, controlled trial.

\begin{abstract}
Materials and Methods-From January 2015 to January 2016, 21 patients with refractory PLP underwent image-guided percutaneous cryoneurolysis procedures. Visual analog scale scores were documented at baseline and 7, 45, and 180 days after the procedure. Responses to a modified Roland Morris Disability Questionnaire were documented at baseline and 7 and 45 days after the procedure.
\end{abstract}

Results-Technical success rate of the procedures was 100\%. There were 6(29\%) minor procedure-related complications. Disability scores decreased from a baseline mean of 11.3 to 3.3 at 45-day follow-up (95\% confidence interval $5.8,10.3 ; P<.0001)$. Pain intensity scores decreased from a baseline mean of 6.2 to 2.0 at long-term follow-up (95\% confidence interval 2.8, 5.6; $P<.0001)$.

Conclusions-Image-guided percutaneous nerve cryoablation is feasible and safe and may represent a new efficacious therapeutic option for patients with phantom pains related to limb loss.

Approximately 185,000 amputations are performed each year in the United States, and studies estimate that nearly 3.6 million people will be living with limb loss worldwide by $2050(1,2)$. A common complaint among amputees is phantom limb pain (PLP), a phenomenon in which unpleasant sensations, often painful and severe, are perceived to originate from an absent limb. PLP occurs in 50\%-80\% of amputees, and there is no consistent evidence to support any specific pain control modality or intervention in this setting (1,3-7). The objectives of this study were to (a) evaluate the safety and feasibility of

\footnotetext{
Address correspondence to J.D.P.; john.david.prologo@emory.edu.

None of the authors have identified a conflict of interest.

From the SIR 2016 Annual Meeting.

Appendices A and B, Figures E1-E5, and Tables E1 and E2 are available online at www.jvir.org.
} 
a separated 2-step procedural methodology consisting of computed tomography (CT)guided perineural diagnostic injection and cryoablation in the setting of refractory PLP; $(b)$ confirm the reproducibility of treatment response in relation to the findings of previous studies; and (c) derive estimates for key study parameters, such as recruitment rates, potentially confounding variables, and the treatment effect and its variation to inform the planning and design of a larger, parallel-armed, randomized controlled clinical trial investigating the efficacy of cryoablation for the treatment of PLP.

\section{MATERIALS AND METHODS}

This study was approved by the university institutional review board and complied with the Health Insurance Portability and Accountability Act. The trial was registered before patient enrollment on ClinicalTrials.gov (NCT02366832).

Subjects

Subjects were recruited from January 2015 to January 2016 from local vascular surgery, orthopedic surgery, and physical medicine and rehabilitation practices. All patients reported phantom pains and/or unwanted sensations related to a previously amputated upper or lower limb that were refractory to conventional treatment (eg, mirror therapy, physical therapy, anxiolytic medications, antidepressant medications, opioids, external cream application, $\gamma$ aminobutyric acid agonists) for at least 6 months. To undergo cryoablation, each patient was required to have documented symptomatic relief from an image-guided perineural injection of bupivacaine and betamethasone. Demographic details for the cohort are listed in Table 1, and inclusion and exclusion criteria are listed in Table 2.

\section{Interview}

During initial consultation, each patient was questioned regarding the distribution and severity of their phantom pains as well as the presence or absence of residual limb pain (RLP). Each symptom complex was classified according to the algorithm presented in Figure E1 (available online at www.jvir.org), and target nerves were identified (Figs E2, E3 [available online at www.jvir.org]).

\section{Block}

Serial axial unenhanced CT images of the residual limb were obtained before any intervention. If a neuroma was identified along the expected course of the offending nerve, that location was the default target. The nerves in question also were evaluated with ultrasound (Fig 1a, b). The patients were positioned in the prone or decubitus position on the CT table, depending on the nerve to be targeted. After obtaining subcutaneous and tract anesthesia with $1 \%$ lidocaine (Hospira, Inc, Lake Forest, Illinois), a 22-gauge spinal needle was advanced under $\mathrm{CT}$ or ultrasound guidance to the target nerve or neuroma. Needle position was confirmed with fluoroscopic $\mathrm{CT}$. Injections of $4 \mathrm{~mL}$ of $0.25 \%$ bupivacaine (Hospira, Inc) and $6 \mathrm{mg}$ of betamethasone (Schering-Plough Corp, Kenilworth, New Jersey) were performed. The patient was monitored for 2 hours after the injection and then discharged. During the subsequent 24 hours, the patients were asked whether or not there

J Vasc Interv Radiol. Author manuscript; available in PMC 2017 December 28. 
was an improvement in symptoms after the injection, and a binary response was recorded as either "yes" or "no."

\section{Ablation}

All ablation procedures were performed under conscious sedation induced with intravenous midazolam (Hospira, Inc) and fentanyl (West-Ward Pharmaceuticals Corp, Eatontown, New Jersey). Using technique and targeting analogous to the diagnostic injections, a 17-gauge Ice-Sphere cryoablation needle (Galil Medical, Inc Arden Hills, Minnesota) was advanced under imaging guidance to the previously identified nerve and/or neuroma. When position was confirmed, the probe temperature was decreased to $-40^{\circ} \mathrm{C}$ over 10 minutes using the Galil Visual-ICE system. Images were obtained and reviewed 8 minutes into the freeze. A passive thaw cycle was then undertaken for 5 minutes, followed by a second 10-minute freeze and subsequent final 5-minute passive thaw (Figs 2 and 3a-d).

\section{Endpoints}

Safety and Feasibility-Safety was measured by the proportion of patients who experienced cryoablation procedure events. Additional safety metrics included nerve block procedure-related adverse events, adverse events (AEs), and serious adverse events (SAEs). AEs included any nonminor complications of ablation as defined by clinical signs or symptoms of infection (fever), hemorrhage (pain, imaging changes), nerve pain worsening, or toxicity of any kind (confusion, seizure), whereas SAEs included life-threatening AEs and death. Because of the pilot nature of the study, it was determined that any AE or SAE reported would necessitate immediate cessation of subject enrollment, execution of a mandatory comprehensive safety review, and notification of the institutional review board.

Feasibility was measured by the technical success rate of the nerve block and cryoablation procedures. Technical success of the nerve block was determined through evaluation of the needle position at the suspected site of pathology. For the cryoablation procedure, technical success was defined as coverage of the neuroma or distal end of the target nerve by the ablation zone under image guidance, with partial or incomplete ablation considered a failure (8).

Efficacy and Patient Perception-Pain intensity and functional status of patients were evaluated before the ablation, 7 days after the ablation, and 45 days after the ablation. In addition, a final, long-term follow-up assessment was performed after the 45-day visit during which patients rated their pain and reported whether or not they would undergo the cryoablation procedure again if given the chance. Four patients who responded affirmatively volunteered to undergo cryoablation again, and data from their second ablation underwent separate analysis.

Ratings of perceived pain intensity were captured using a visual analog scale (VAS) (Fig E4 [available online at www.jvir.org]) (9). The VAS is a 100-mm horizontal line with word descriptors at 2 opposing extremes at either end. A patient completes the VAS by making a mark at the point along the continuum that represents his or her intensity of pain, with $0 \mathrm{~mm}$

J Vasc Interv Radiol. Author manuscript; available in PMC 2017 December 28. 
$=$ no pain and $100 \mathrm{~mm}=$ worst possible pain. The VAS is scored by measuring the distance in millimeters from the patient's mark to 1 of the ends of the line.

Subject functional status was evaluated with a modified Roland Morris Disability Questionnaire (RMDQ). The RMDQ is a health status measure originally designed to assess physical disability related to back pain $(10,11)$. The RMDQ consists of 24 statements describing activity limitations resulting from back pain to which patients answer with either "yes," equal to 1 point, or "no," corresponding to zero, for a total of 24 possible points (12). A minimum score of zero corresponds to no disability, whereas a maximum score of 24 corresponds to maximum disability. For the purposes of this study, the RMDQ was adapted to focus on PLP (Appendix A [available online at www.jvir.org]).

\section{Statistical Analysis}

A multilevel (mixed-effects) linear growth modeling approach was used to evaluate changes in efficacy variables over time, while accounting for the clustering of data within patients. Multilevel linear growth modeling was uniquely suitable for the present study, as it permits (a) the responses to treatment to vary heterogeneously both over time and between individual patients, $(b)$ the number of assessments and time between assessments to be distinct for each individual, and (c) accurate estimation in the presence of missing data (1315). Changes in efficacy endpoints in response to cryoablation were analyzed using a 2-level model (level 1: visit; level 2: patient) with the variable for visit (baseline/day 0, day 7, day 45 , long-term follow-up) included both as a fixed effect and as a random effect at the patient level.

To evaluate differences in treatment responses between procedures in patients who underwent a second ablation, the model was modified into a 3-level model (level 1: visit; level 2: procedure; level 3: patient) with the addition of a dummy variable for procedure $(0=$ first procedure, $1=$ second procedure) and its interaction with the visit variable as fixed effects; an additional random effect for procedure was included at the patient level, whereas the random effect for visit was moved to the procedure level. Because of the longitudinal repeated measurements on the same subject and the unequal spacing between visit 3 and the long-term follow-up assessment, a heterogeneous Toeplitz variance-covariance structure was used to account for the correlated errors and heterogeneous variances for each visit.

To compare the difference in response to treatment, the model included continuous covariates, such as the patient's age and the number of years since amputation, and dummy variables representing the comorbid pain condition and patient demographic variables collected during the screening interview as fixed factors; a dummy variable representing whether the patient underwent a second ablation or not $(1=$ reablation, $0=$ no reablation $)$ was also included. Model fitting procedures began with the full model, which included all the explanatory variables, their interactions with the visit (time) variable, and a 3-way interaction between visit and the dummy variables for RLP and neuroma, to account for potential changes in response that varied with time and specific combinations of pain comorbidities. Selection of the final model was achieved through (a) comparisons of the Bayesian information criterion, which was calculated using the number of patients included in the model instead of the number of observations to account for clustering, and (b) 
evaluation of likelihood ratio tests (16). Insignificant predictors were excluded from the model in the final analyses reported for each endpoint.

Values summarized using descriptive statistics are reported as mean $\pm \mathrm{SD}$, whereas values estimated using statistical models are reported as mean (lower 95\% confidence interval bound, upper $95 \%$ confidence interval bound). For all reported analyses, multilevel models were fit using restricted maximum likelihood estimation, and to account for the small sample size, calculations of degrees of freedom were made using the Kenward-Roger method (17); likelihood ratio tests employed in the selection of fixed effects used values obtained using maximum-likelihood estimation. All statistical analyses were performed using SAS/STAT software, version 9.3 (SAS Institute Inc, Cary, North Carolina) using an a of .05.

\section{RESULTS}

During the year-long enrollment period, 24 patients were recruited corresponding to a recruitment rate of 1.75 patients per month or 7 patients every 4 months. All 24 patients met the eligibility criteria evaluated in the screening interview and received nerve block injections. During the evaluation period after the injection, 3 patients were excluded because of a lack of response, and the remaining patients $(n=21)$ underwent cryoablation.

All patients were evaluated 7 days and 45 days after the procedure. The long-term follow-up assessment occurred at a mean of 194 days \pm 99 after the procedure. One patient died during the period between visit 3 and the long-term follow-up assessment and was considered lost to follow-up.

\section{Endpoints}

Safety and Feasibility—All procedures were technically successful—all target nerves and/or neuromas were successfully ablated. There was 1 SAE consisting of a patient's death, which was concluded to be unrelated to treatment or participation in this study. Class B minor complications occurred in 6 (29\%) patients comprising procedure-related pain during the initial days after the procedure that required additional antiinflammatory prescription medication (18).

Pain Intensity, Functional Status, and Patient Perceptions-Mean pain intensity scores and functional status ratings at each visit are listed in Table 3 and illustrated in Figure 4. At day 7 after the procedure, pain intensity scores had decreased by a mean of $0.8(-0.17$, 1.7) points from baseline; however, this change was not statistically significant. Between 7 days and 45 days after the procedure, pain intensity decreased by a mean of $3.1(2.2,4.0)$ points $(P<.0001)$, corresponding to a statistically significant overall reduction of 3.9 (2.9, 4.9) points $(P<.0001)$ compared with baseline. At the long-term follow-up assessment, changes in pain intensity scores compared with 45 days after the procedure were not significantly different; however, compared with baseline, there was a statistically significant $(P<.0001)$ overall reduction in mean pain intensity of $4.2(2.8,5.6)$ points. Ratings of functional status decreased between baseline and day 7 after the procedure by a mean of 2.0 $(-1.0,4.9)$ points; however, this change was not statistically significant. At 45 days after the procedure, ratings of functional status had decreased significantly $(P<.0001)$ by a mean of 
$6.1(4.0,8.2)$ points compared with 7 days after the procedure. Compared with baseline, there was a statistically significant $(P<.0001)$ overall reduction in functional status ratings at 45 days after the procedure corresponding to a mean of $8.0(5.8,10.3)$ points. Coefficient estimates for fixed and random effects for pain intensity and functional status analyses are listed in Table 4.

To identify explanatory variables that affect changes in pain intensity scores and functional status ratings, cofactors for comorbid pain conditions, patient demographics variables, and potential time-varying covariates were included in the model as fixed effects. Significant predictors were found for pain intensity ratings. The extremity of amputation (upper vs lower limb), whether the patient underwent repeat ablation, and whether the patient had RLP were significant predictors for pain intensity ratings. Patients with upper limb amputations had a higher mean pain intensity rating than patients with lower limb amputations by 1.1 $(0.5,1.7)$ points $(P=.0014)$, and patients who underwent repeat ablation had a mean pain intensity score at baseline (first procedure) that was $4.2(3.5,4.8)$ points higher $(P<.0001)$ than patients who underwent only 1 ablation. Patients without RLP had a significantly higher mean pain intensity than patients with RLP by $3.0(1.6,4.4)$ points $(P=.0003)$ and had significantly greater overall reductions in pain intensity from baseline of $2.5(0.8,4.2)$ points $(P=.0066)$ at the day-45 visit and $4.4(2.4,6.3)$ points $(P=.0002)$ compared with patients with RLP. The final mean pain intensity ratings at the long-term follow-up assessment was $3.1(2.5,3.6)$ points for patients without RLP and $4.4(3.8,5.1)$ points for patients with RLP, corresponding to a statistically significant difference of $1.4(0.5,2.2)$ points $(P=.0028)$. Cumulatively, these results indicated that RLP had a significant impact on the change in pain intensity over time such that patients with RLP had lower baseline and lesser overall changes over time in pain intensity ratings compared with patients without RLP, which had higher baseline and greater overall changes over time in pain intensity ratings.

At the long-term follow-up assessment, patients were asked if they would undergo the procedure again. Of the 21 patients who underwent successful cryoablations, 1 patient (5\%) was deceased at the time of the long-term follow-up assessment, 4 patients (19\%) volunteered to undergo a second cryoablation, and 14 of the remaining 16 patients (67\%) responded affirmatively. Considering only patients who underwent a single cryoablation, $90 \%$ responded that they would undergo the procedure again.

\section{Patients Who Underwent Second Ablation}

A second cryoablation procedure was performed in 4 patients. A second nerve block injection was not performed. Cryoablation was performed successfully the second time in all 4 patients (100\%). The long-term follow-up assessment occurred at a mean of 165 days \pm 73 after the second ablation procedure, and there were no reports of AEs throughout the followup period.

Changes in pain intensity and functional status over time were evaluated and compared with the first procedure (Table 5); there were no statistically significant differences in functional status between the 2 procedures either at each time point or over time. Patients had a mean pain intensity score at baseline of the first procedure that was a mean of $2.0(0.6,3.4)$ points 
greater $(P=.0111)$ than at baseline of the second procedure, indicating that despite their desire to undergo a second ablation procedure, an overall improvement in pain intensity scores remained from the first ablation procedure. There were no differences in overall changes in pain intensity scores over time between the first and second procedure, indicating that patients who underwent repeat ablation had consistent responses to treatment between the 2 ablation procedures. At the long-term follow-up assessment after the second cryoablation, pain intensity scores in patients with reablation had significantly decreased by a mean of $4.25(2.1,6.4)$ points $(P=.0013)$ compared with baseline of the first procedure. Despite experiencing a statistically significant reduction in pain intensity scores after both cryoablation procedures, when patients who underwent reablation were asked if they would undergo the procedure again, only 1 patient $(25 \%)$ responded yes.

Incorporation of comorbid pain conditions, patient demographics variables, and potential time-varying covariates revealed a significant difference in pain intensity scores associated with RLP; no significant predictors were found for functional status. Patients with RLP had a significantly lower pain intensity rating at the long-term follow-up assessment of the second procedure compared with the first procedure by $2.7(0.5,4.9)$ points $(P=.0209)$. However, compared with patients without RLP, there were no statistically significant differences in mean pain intensity ratings between the 2 procedures both at each visit and from baseline over time.

\section{DISCUSSION}

Amputation results in multidirectional reorganization and sensitization of neural signaling from transected peripheral somatosensory afferent fibers to the cortex. PLP-pain perceived to originate from an absent body part after amputation-represents the symptomatic end manifestation of ectopic inputs along this transformed pathway (19-21). Numerous studies have reported on interventions at different points along this altered pathway, from direct and indirect cortical modulation to surgical resection of the distal residual nerve (and at many points in between), yet no accepted guidelines for treatment have emerged (21-26). Treatment strategies targeting the peripheral nerve, such as catheter placement for infusion of anesthetic postoperatively, peripheral neuromodulation, lidocaine injections, and cryoanalgesia guided by nerve stimulation, have been reported more recently as potentially efficacious (27-30).

Support for peripheral intervention is rooted in well-known changes after amputation-related nerve transection. Plasticity and "windup" related to peripheral nerve scar tissue traction, compression of residual nerves, ischemia, or peripheral upregulation of ectopic ion channels contributes to unpleasant sensations that localize to the deafferented body part (31). The microenvironment around a peripheral axotomy induces biochemical changes that result in increased expression of voltage-sensitive sodium channels, decreased potassium channel expression, altered transduction molecules involved in mechanosensitivity and heat and cold sensitivity, increased concentrations of inflammatory mediators, and altered adrenoreceptor subtype expression - the end product of which are ectopic action potentials $(21,29,32-34)$. These "firings" have been characterized and implicated in the establishment of ongoing noxious signals, intensification, and summation effects on ectopic signals from the dorsal 
root ganglion, central nervous system reorganization, and global neuraxis sensitization, not to mention the pain itself $(19,20,25,35)$. Further reasoning behind the pursuit of treatment options that target the peripheral nervous system can be found in reports correlating RLP with PLP, the exacerbation of PLP after mechanical stimulation of the peripheral nerve or neuroma, and improvement of symptoms after surgical resection of neuromas $(19,22,36)$.

Mechanistically, cryoablation affects nerves specifically through (a) ice crystal-mediated vasa vasorum damage and endoneural edema, $(b)$ wallerian degeneration, $(c)$ direct physical injury to axons, and (d) dissolution of microtubules resulting in cessation of axonal transport $(29,37-39)$. The end result of these routes of neuronal damage is decreased pain sensation as a result of conduction cessation, activation of descending inhibition, blockade of excitatory transmitter systems, or generalized sodium channel blockade (40).

Clinically, cryoanalgesia has been used safely in 2 small series to treat PLP and RLP associated with stump neuromas $(29,41)$. Moesker et al (29) retrospectively reviewed and reported 5 cases of nerve stump cryoneurolysis with follow-up to at least 2.5 years. Three of the 5 patients reported $90 \%-100 \%$ resolution of their symptoms, 1 patient reported $40 \%$ improvement, and 1 patient reported 20\% improvement. Procedurally, the nerves were located with neurostimulation, and the freeze-thaw cycles $(n=2)$ were performed at 3 minutes (freeze) and 2 minutes (thaw). There were no procedure-related complications. Neumann et al (41) reported their experience placing cryoprobes with electrophysiologic guidance in patients with refractory stump pain. The freeze-thaw cycle in this study was 60 seconds (freeze) and 60 seconds (freeze), and the number of cycles depended on the patient response, up to a maximum of 5 . At 3 months, 9 of the patients reported pain relief, and 3 of the 9 patients reported continued relief at 12 months.

In the present study, the technical success rate of all procedures was $100 \%$. There were no reports of AEs throughout the follow-up periods after either the cryoablation procedure or diagnostic nerve block. There was 1 SAE consisting of a patient's death, which was concluded to be unrelated to treatment or the patient's participation in the study. During the initial days after the procedure, 6 patients (29\%) experienced pain that required additional antiinflammatory prescription medication.

As reported in previous studies, reductions in baseline pain intensity scores were observed after cryoablation. Pain intensity scores were significantly reduced to $37.1 \%$ and $32.3 \%$ of the baseline mean pain intensity score at assessment 45 days after the procedure and at the long-term follow-up assessment, respectively. Analysis of 4 patients who underwent reablation revealed a lasting reduction in pain intensity scores from the first ablation procedure and a consistent reduction in pain intensity scores between the 2 ablation procedures.

Patients with upper limb amputations had greater mean pain intensity scores compared with patients with lower limb amputations, and patients with RLP had lower baseline and lesser overall improvements over time in pain intensity ratings compared with patients without RLP. Similarly, patients with RLP after reablation presented with higher baseline pain intensity scores; however, there were no statistically significant differences in mean pain 
intensity scores at each follow-up visit and from baseline over time between the 2 procedures.

Using the RMDQ, improvements in functional status ratings were observed after both cryoablation procedures. In contrast to pain intensity scores, no explanatory variables were found to influence functional status ratings either at each follow-up visit or over time. In patients with reablation, functional status ratings both at each follow-up visit and over time were not significantly different. Although these results indicate a consistent response to treatment between the 2 cryoablation procedures, they also imply that before undergoing reablation, functional status ratings in these patients had returned to levels before treatment.

This pilot study was conducted to evaluate the use of image-guided cryoablation in a single cohort of patients with refractory PLP. A 2-step methodology was used, which consisted of a diagnostic nerve block injection followed by cryoablation that occurred on separate occasions. For the purpose of designing such a trial, separation of the block and ablation procedures is advantageous because it allows for patients' response to treatment to be evaluated before an ablation is performed as well as the incorporation of a parallel control group that receives either a sham procedure or active control (eg, second nerve block injection); however, this benefit comes with the cost of doubling the opportunities for procedure-related complications and adverse events and potentially affecting a patient's response to treatment in an unknown way.

Over the year-long recruitment period, 1.75 patients per month, or 7 patients every 4 months, were recruited. The percentage of lost patients, which included the 3 patients with nerve block failures and 1 patient who died, was $16.7 \%$. Together, these metrics demonstrate acceptable recruitment and retention rates. A brief illustration of the results of this study will be used to inform the planning and sample size calculations of a larger, parallel-armed, randomized controlled clinical trial and is provided in Appendix B, Figure E5, and Tables E1 and E2 (available online at www.jvir.org) (42).

This study is limited by its exploratory nature (single-arm pilot cohort with no use of control, randomization, or blinding) and variability at the final follow-up point. In addition, medication logs were not collected, and the subjective reporting of a quantitative pain score or binary response to functional outcome questions ultimately captures only a portion of the impact of novel interventions for pain. Measurement tools with wider spectra of interrogation, such as the Yale Multidisciplinary Pain Inventory, would have allowed for a more complete analysis $(43,44)$.

In conclusion, percutaneous image-guided cryoneurolysis for treatment of PLP was safe and feasible. This approach may represent a new efficacious therapeutic option for patients with phantom pains related to limb loss.

\section{Supplementary Material}

Refer to Web version on PubMed Central for supplementary material. 


\section{Acknowledgments}

J.D.P. has had research funded by Galil Medical, Inc. (Arden Hills, Minnesota).

The study coordinator was Maria Rivas, MD. The study sponsor was Galil Medical, Inc (Arden Hills, Minnesota).

\section{ABBREVIATIONS}

$\begin{array}{ll}\text { AE } & \text { adverse event } \\ \text { PLP } & \text { phantom limb pain } \\ \text { RLP } & \text { residual limb pain } \\ \text { RMDQ } & \text { Roland Morris Disability Questionnaire } \\ \text { SAE } & \text { serious adverse event } \\ \text { VAS } & \text { visual analog scale }\end{array}$

\section{References}

1. Ziegler-Graham K, MacKenzie EJ, Ephraim PL, Travison TG, Brookmeyer R. Estimating the prevalence of limb loss in the United States: 2005 to 2050. Arch Phys Med Rehabil. 2008; 89:422429. [PubMed: 18295618]

2. Owings MF, Kozak LJ. Ambulatory and inpatient procedures in the United States, 1996. Vital Health Stat. 1998; 13:1-119.

3. Ephraim PL, Wegener ST, MacKenzie EJ, Dillingham TR, Pezzin LE. Phantom pain, residual limb pain, and back pain in amputees: results of a national survey. Arch Phys Med Rehabil. 2005; 86:1910-1919. [PubMed: 16213230]

4. Rothgangel A, Braun S, de Witte L, Beurskens A, Smeets R. Development of a clinical framework for mirror therapy in patients with phantom limb pain: an evidence-based practice approach. Pain Pract. 2016; 16:422-434. [PubMed: 25880456]

5. Department of Veterans Affairs; Department of Defense. [Accessed October 19, 2016] Va/DoD clinical practice guideline for rehabilitation of lower limb amputation. 2008. Available at: http:// www.healthquality.va.gov/amputation/amp_sum_508.pdf

6. Schley MT, Wilms P, Toepfner S, et al. Painful and nonpainful phantom and stump sensations in acute traumatic amputees. J Trauma. 2008; 65:858-864. [PubMed: 18849803]

7. Richardson C, Glenn S, Nurmikko T, Horgan M. Incidence of phantom phenomena including phantom limb pain 6 months after major lower limb amputation in patients with peripheral vascular disease. Clin J Pain. 2006; 22:353-358. [PubMed: 16691088]

8. Goldberg SN, Grassi CJ, Cardella JF, et al. Image-guided tumor ablation: standardization of terminology and reporting criteria. Radiology. 2005; 235:728-739. [PubMed: 15845798]

9. Melzack R. The McGill Pain Questionnaire: major properties and scoring methods. Pain. 1975; 1:277-299. [PubMed: 1235985]

10. Roland M, Morris R. A study of the natural history of back pain. Part I: development of a reliable and sensitive measure of disability in low-back pain. Spine (Phila Pa 1976). 1983; 8:141-144. [PubMed: 6222486]

11. Roland M, Morris R. A study of the natural history of low-back pain. Part II: development of guidelines for trials of treatment in primary care. Spine (Phila Pa 1976). 1983; 8:145-150. [PubMed: 6222487]

12. Rocchi MB, Sisti D, Benedetti P, Valentini M, Bellagamba S, Federici A. Critical comparison of nine different self-administered questionnaires for the evaluation of disability caused by low back pain. Eura Medicophys. 2005; 41:275-281. [PubMed: 16474281] 
13. Diez-Roux AV. Multilevel analysis in public health research. Annu Rev Public Health. 2000; 21:171-192. [PubMed: 10884951]

14. Gewandter JS, et al. Reporting of missing data and methods used to accommodate them in recent analgesic clinical trials: ACTTION systematic review and recommendations. Pain. 2014; 155:1871-1877. [PubMed: 24993384]

15. Sturgeon JA, Zautra AJ, Arewasikporn A. A multilevel structural equation modeling analysis of vulnerabilities and resilience resources influencing affective adaptation to chronic pain. Pain. 2014; 155:292-298. [PubMed: 24120460]

16. Pauler DK. The Schwarz criterion and related methods for normal linear models. Biometrika. 1998; 85:13-27.

17. Kenward MG, Roger JH. Small sample inference for fixed effects from restricted maximum likelihood. Biometrics. 1997; 53:983-997. [PubMed: 9333350]

18. Sacks D, McClenny TE, Cardella JF, Lewis CA. Society of Interventional Radiology clinical practice guidelines. J Vasc Interv Radiol. 2003; 14(9 Pt 2):S199-S202. [PubMed: 14514818]

19. Flor H. Phantom-limb pain: characteristics, causes, and treatment. Lancet Neurol. 2002; 1:182189. [PubMed: 12849487]

20. Griffin SC, Tsao JW. A mechanism-based classification of phantom limb pain. Pain. 2014; 155:2236-2242. [PubMed: 24857794]

21. Subedi B, Grossberg GT. Phantom limb pain: mechanisms and treatment approaches. Pain Res Treat. 2011; 2011:864605. [PubMed: 22110933]

22. Weeks SR, Anderson-Barnes VC, Tsao JW. Phantom limb pain: theories and therapies. Neurologist. 2010; 16:277-286. [PubMed: 20827116]

23. Wolff A, Vanduynhoven E, van Kleef M, Huygen F, Pope JE, Mekhail N. 21. Phantom pain. Pain Pract. 2011; 11:403-413. [PubMed: 21447079]

24. Pirowska A, Wloch T, Nowobilski R, Plaszewski M, Hocini A, Ménager D. Phantom phenomena and body scheme after limb amputation: a literature review. Neurol Neurochir Pol. 2014; 48:5259. [PubMed: 24636771]

25. Foell J, Bekrater-Bodmann R, Flor H, Cole J. Phantom limb pain after lower limb trauma: origins and treatments. Int J Low Extrem Wounds. 2011; 10:224-235. [PubMed: 22184752]

26. Foell J, Bekrater-Bodmann R, Diers M, Flor H. Mirror therapy for phantom limb pain: brain changes and the role of body representation. Eur J Pain. 2014; 18:729-739. [PubMed: 24327313]

27. Cornish P, Wall C. Successful peripheral neuromodulation for phantom limb pain. Pain Med. 2015; 16:761-764. [PubMed: 25138543]

28. Borghi B, D'Addabbo M, White PF, et al. The use of prolonged peripheral neural blockade after lower extremity amputation: the effect on symptoms associated with phantom limb syndrome. Anesth Analg. 2010; 111:1308-1315. [PubMed: 20881281]

29. Moesker AA, Karl HW, Trescot AM. Treatment of phantom limb pain by cryoneurolysis of the amputated nerve. Pain Pract. 2014; 14:52-56. [PubMed: 23279331]

30. Chabal C, Jacobson L, Russell LC, Burchiel KJ. Pain response to perineuromal injection of normal saline, epinephrine, and lidocaine in humans. Pain. 1992; 49:9-12. [PubMed: 1594285]

31. Rajput K, Reddy S, Shankar H. Painful neuromas. Clin J Pain. 2012; 28:639-645. [PubMed: 22699131]

32. Flor H, Nikolajsen L, Staehelin Jensen T. Phantom limb pain: a case of maladaptive CNS plasticity? Nat Rev Neurosci. 2006; 7:873-881. [PubMed: 17053811]

33. Buonocore M. Where is hidden the ghost in phantom sensations? World J Clin Cases. 2015; 3:542544. [PubMed: 26244147]

34. Drummond PD. Neuronal changes resulting in up-regulation of alpha-1 adrenoceptors after peripheral nerve injury. Neural Regen Res. 2014; 9:1337-1340. [PubMed: 25221588]

35. Borghi B, D'Addabbo M, Borghi R. Can neural blocks prevent phantom limb pain? Pain Manag. 2014; 4:261-266. [PubMed: 25300383]

36. Ducic I, Mesbahi AN, Attinger CE, Graw K. The role of peripheral nerve surgery in the treatment of chronic pain associated with amputation stumps. Plast Reconstr Surg. 2008; 121:908-914. discussion 915-917. [PubMed: 18317139] 
37. Wagner R, DeLeo JA, Heckman HM, Myers RR. Peripheral nerve pathology following sciatic cryoneurolysis: relationship to neuropathic behaviors in the rat. Exp Neurol. 1995; 133:256-264. [PubMed: 7649230]

38. Myers RR, Heckman HM, Powell HC. Axonal viability and the persistence of thermal hyperalgesia after partial freeze lesions of nerve. J Neurol Sci. 1996; 139:28-38. [PubMed: 8836969]

39. Myers RR, Powell HC, Heckman HM, Costello ML, Katz J. Biophysical and pathological effects of cryogenic nerve lesion. Ann Neurol. 1981; 10:478-485. [PubMed: 7305300]

40. Zhou L, Shao Z, Ou S. Cryoanalgesia: electrophysiology at different temperatures. Cryobiology. 2003; 46:26-32. [PubMed: 12623025]

41. Neumann V, O'Connor RJ, Bush D. Cryoprobe treatment: an alternative to phenol injections for painful neuromas after amputation. AJR Am J Roentgenol. 2008; 191:W313. author reply W314. [PubMed: 19020222]

42. Guo Y, Logan HL, Glueck DH, Muller KE. Selecting a sample size for studies with repeated measures. BMC Med Res Methodol. 2013; 13:100. [PubMed: 23902644]

43. Kerns RD, Turk DC, Rudy TE. The West Haven-Yale Multidimensional Pain Inventory (WHYMPI). Pain. 1985; 23:345-356. [PubMed: 4088697]

44. Turk DC, Dworkin RH, Allen RR, et al. Core outcome domains for chronic pain clinical trials: IMMPACT recommendations. Pain. 2003; 106:337-345. [PubMed: 14659516]

\section{APPENDIX A. ADAPTED ROLAND MORRIS DISABILITY QUESTIONNAIRE Disability Rating Scale for Pain}

When you have pain, you may find it difficult to do some of the things you normally do. This list contains some sentences that people have used to describe themselves when they have pain. When you read them, you may find that some stand out because they describe you today. As you read the list, think of yourself today. When you read a sentence that describes you today, make a check mark next to it. If the sentence does not describe you, do not mark it and go on to the next one. Remember, only mark the sentence if you are sure that it describes you today.

1. I stay at home most of the time because of the pain.

2. I change position frequently to try to be more comfortable.

3. I move more slowly than usual because of the pain.

4. Because of the pain, I am not doing any of the jobs that I usually do around the house.

5. Because of the pain, I use extra help to get around.

6. Because of the pain, I lie down to rest more often.

7. Because of the pain, I am less interested in prosthetics.

8. Because of the pain, I ask other people to do things for me.

9. I get dressed more slowly than usual because of the pain.

10. I stand up for only short periods of time because of the pain.

11. Because of the pain, I try not to move much.

12. I find it difficult to get around because of the pain. 
13. My leg/arm hurts most of the time.

14. I find it difficult to turn over in bed because of the pain.

15. My appetite is not very good because of the pain.

16. I have trouble putting on my socks (or stockings) because of the pain.

17. I walk only short distances because of the pain.

18. I sleep less because of the pain.

19. Because of the pain, I get dressed with help from someone else.

20. I sit down for most of the day because of the pain.

21. I avoid jobs around the house because of the pain.

22. Because of the pain, I am more irritable and bad tempered with people.

23. I stay in bed most of the time because of the pain.

\section{APPENDIX B. SAMPLE SIZE CALCULATIONS}

Sample size estimation was performed for a larger, parallel-armed, sham-controlled, randomized clinical trial investigating changes in pain intensity scores, as measured using the VAS, between patients undergoing cryoablation and patients receiving a sham procedure. Patients were assumed to match the same inclusion and exclusion criteria as used in the pilot study. VAS score data were planned to be analyzed using a 2-level linear growth model with fixed effects of visit (visit 1/baseline, visit 2, visit 3, visit 4), treatment group (cryoablation, sham), and a visit*group interaction term and random effects for patient (level 2) and visit (level 1), in addition to a random coefficient for visit at the patient level.

It was assumed that the primary interest will be to investigate differences in changes in VAS scores (pain intensity) over time between patients who undergo cryoablation compared with patients who receive a sham procedure. In the multilevel growth model, this corresponds to an interaction term between treatment and visit. Based on the pilot trial results, it is hypothesized that after 6 months, there will be a minimum difference of 0.5 points between the changes in VAS score over time in the cryoablation and sham groups:

$$
\begin{aligned}
& H_{o}:\left(\Delta V A S_{\text {Cryo }}-\Delta V A S_{\text {sham }}\right) \leq 0.5 \\
& H_{A}:\left(\Delta V A S_{\text {Cryo }}-\Delta V A S_{\text {Sham }}\right)>0.5
\end{aligned}
$$

Where $\triangle V A S_{C r y o}$ is the change (visit 4 - visit 1/baseline) in VAS score over time for the cryoablation group, $\triangle V A S_{S h a m}$ is the change in VAS score over time for the sham group, and 0.5 is the minimum difference between VAS score changes.

Variance and covariance patterns were based off of the results observed in the pilot trial. To obtain conservative estimates, the largest visit variance estimate, 7.1824 ( $\mathrm{SD}=2.6833$ ), was assumed. The correlations among visits were assumed to have the same heterogeneous Toeplitz structure that was observed in the pilot trial (Table E1). With this information, 
GLIMMPSE (http://glimmpse.samplesizeshop.org/) (42) was used to perform sample size calculations for a trial with the following characteristics and assumptions:

- $\quad$ Two-level model: level 1 = visit (time), level $2=$ patient.

- $\quad$ Study duration of 6 months.

- $\quad$ Measurements occurring at baseline and 7 days, 90 days (approximately 3 months), and 180 days (approximately 6 months).

- $\quad$ Treatment and randomization at the patient level (level 2).

- $\quad$ Parallel groups with 1:1 allocation ratio: cryoablation (treatment), sham (control)

- $\quad$ Single amputation per patient assumed.

- $\quad$ Randomization at the person level, performed after a successful nerve block.

- Mean difference in change in VAS score over time between cryoablation and sham groups of 0.5 point per month. To aid in planning, additional mean differences above and below the estimated value should be included $( \pm 10 \%$, $\pm 20 \%$ ).

- $\quad$ The variance of the difference is assumed to be 7.1824. To aid in planning, additional mean differences above and below the estimated value should be included $( \pm 10 \%, \pm 20 \%)$.

- $\quad$ Estimated correlations among measurements with a heterogeneous Toeplitz structure, as listed in Table E1.

- $\quad$ Attrition rate of $16.7 \%$.

- $\quad$ Power $=80 \% ; a=.05$.

Under these assumptions, Table E2 lists the number of patients per group required for the corresponding ranges of mean differences, variance variabilities, and attrition rate. Based on the estimated mean difference in VAS scores over time of 0.5 point and the assumed maximum variance estimate and correlation pattern from the pilot trial, the total sample size required is 60 patients, or 30 patients per group. Assuming an attrition rate of $16.7 \%$, the total sample size required is inflated to 72 patients, or 36 patients per group.

Although conservative estimates and assumptions have been made, an interim analysis was planned to occur after all patients have completed the second follow-up visit (visit 3; approximately 3 months). The goals of interim analyses will be to evaluate the adequacy of the initial estimates and assumptions used for sample size calculations. In addition, sample size reestimation efforts may occur during the interim to ensure that there is a sufficient number of patients to maintain the overall power of $80 \%$. An illustration of the study's flow is provided (Fig E5). 

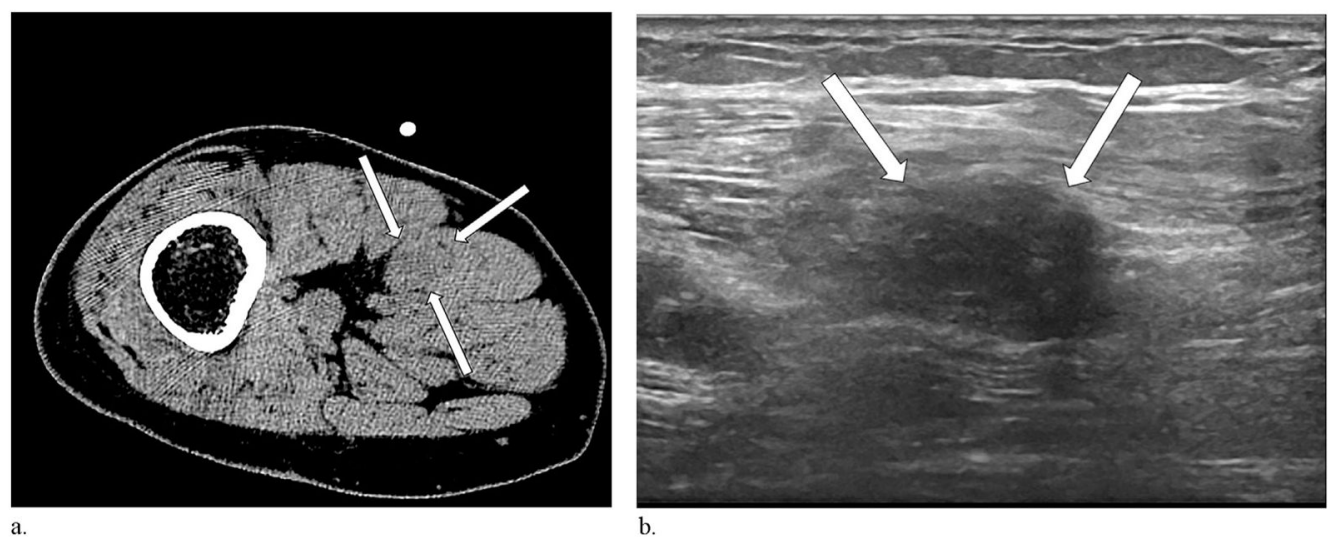

Figure 1.

(a) Single axial CT image of the residual limb in a patient with a traumatic below-the-knee amputation and pains involving the ventral surface and toes of the ipsilateral phantom foot demonstrates a $1.1 \mathrm{~cm} \times 1.5 \mathrm{~cm}$, slightly hypodense enlargement along the course of the sciatic nerve, consistent with a neuroma (arrows). (b) Ultrasound performed at the same time demonstrates a hypoechoic correlate (arrows). 


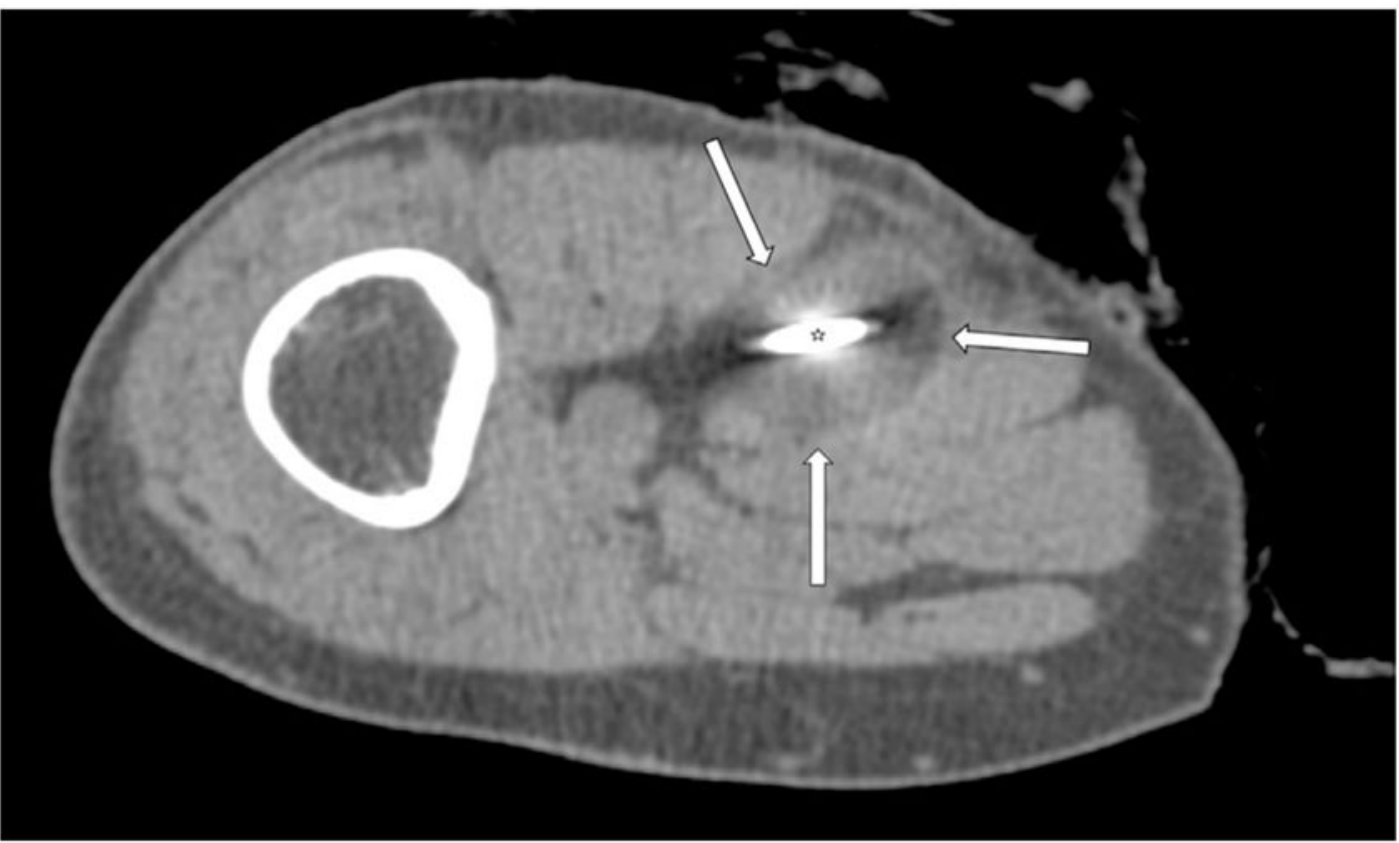

Figure 2.

Single axial CT image obtained 8 minutes into the first freeze-thaw cycle during the cryoablation procedure for the same patient as in Figure 1 demonstrates the cryoprobe (star) and a characteristic hypoattenuating ablation zone (arrows). 

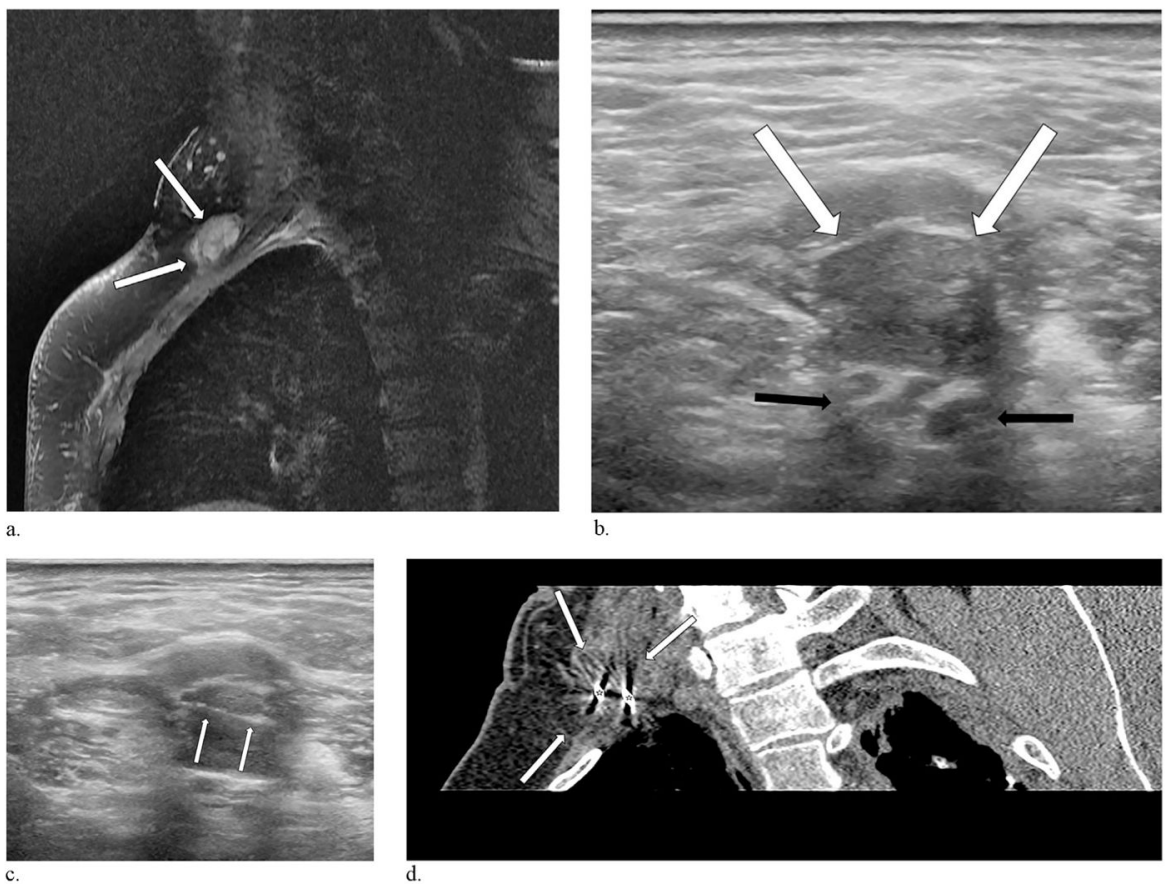

Figure 3.

A 54-year-old man presented with a 9-year history of upper extremity, lightning-like phantom sensations involving his lateral fingers and forearm. (a) Magnetic resonance imaging performed 2 years before the procedure demonstrates a $1.5 \mathrm{~cm} \times 2.6 \mathrm{~cm}$ brachial plexus neuroma (arrows). (b) Ultrasound performed the day of the injection delineates the neuroma (white arrows) adjacent to the brachial plexus cords (black arrows). (c) Ultrasound guidance was employed in this case to place the cryoprobes (arrows) into the neuroma. (d) Reconstructed oblique CT image obtained 8 minutes into the first freeze cycle demonstrates 2 cryoprobes (stars) and the ablation zone engulfing the neuroma (arrows). 


\section{Outcome Variables Following Procedure}

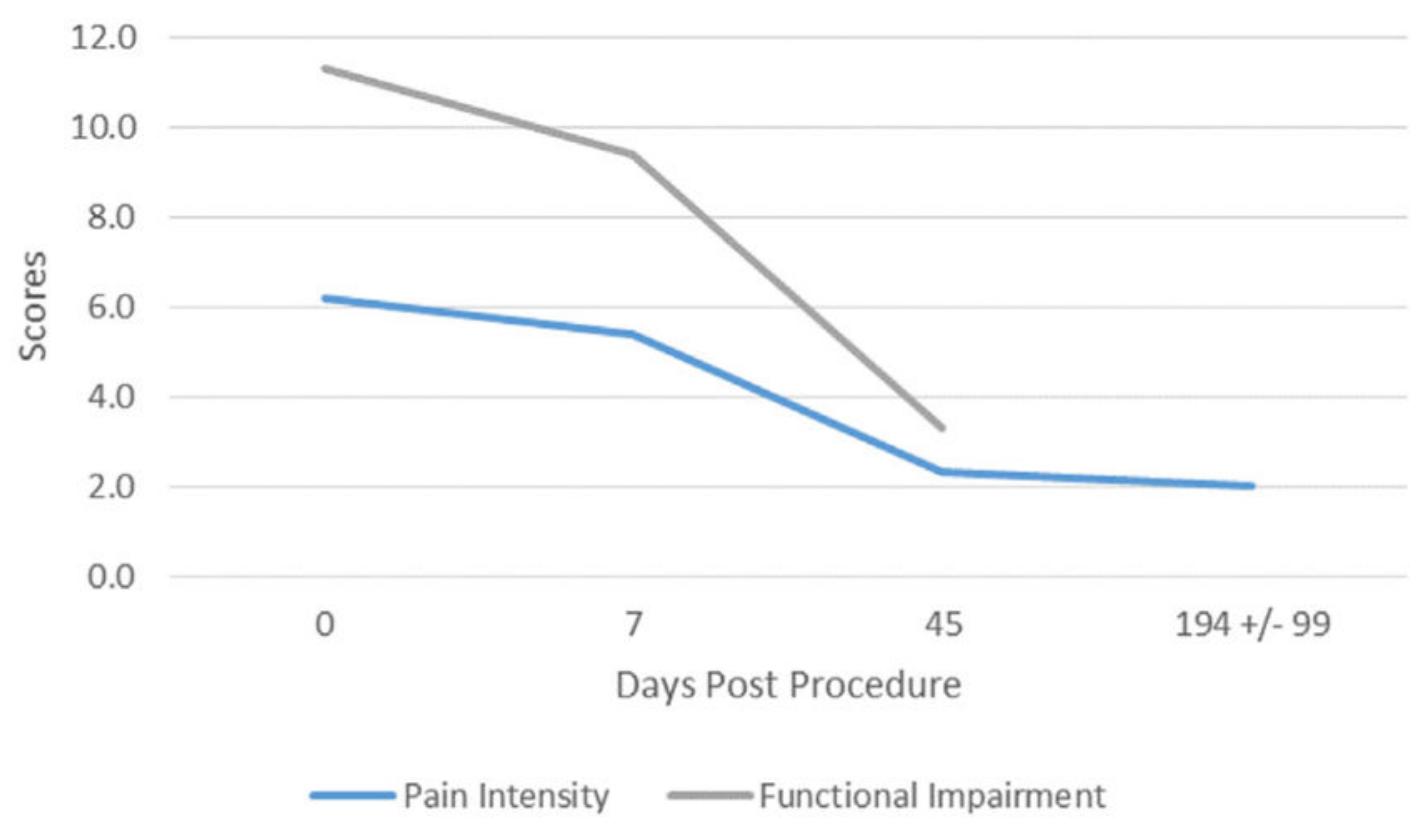

Figure 4.

Mean pain intensity scores and functional status ratings. 


\section{Table 1}

Subject Demographic Data

\begin{tabular}{|c|c|}
\hline Factors & Values \\
\hline Number of patients & 21 \\
\hline Age, y, mean (range) & $56.9(34-91)$ \\
\hline Female sex & $12(60 \%)$ \\
\hline Time since amputation, y, mean (range) & $10.2(1-50)$ \\
\hline Preoperative pain intensity, mean \pm SD & $6.2 \pm 2.2$ \\
\hline \multicolumn{2}{|l|}{ Residual limb pain } \\
\hline Yes & $8(38.1 \%)$ \\
\hline No & $13(61.9 \%)$ \\
\hline \multicolumn{2}{|l|}{ Neuroma } \\
\hline Yes & $13(61.9 \%)$ \\
\hline No & $8(38.1 \%)$ \\
\hline \multicolumn{2}{|l|}{ Site of amputation } \\
\hline Upper extremity & $4(19 \%)$ \\
\hline Above elbow & $1(25 \%)$ \\
\hline Below elbow & $1(25 \%)$ \\
\hline Complete forequarter & $2(50 \%)$ \\
\hline Lower extremity & $17(81 \%)$ \\
\hline Above knee & $10(58.8 \%)$ \\
\hline Below knee & $7(41.2 \%)$ \\
\hline \multicolumn{2}{|l|}{ Reason for amputation } \\
\hline Peripheral vascular disease & $7(33.3 \%)$ \\
\hline Neoplasm & $1(4.8 \%)$ \\
\hline Thrombosis & $2(9.5 \%)$ \\
\hline Trauma & $9(42.9 \%)$ \\
\hline Tumor & $2(9.5 \%)$ \\
\hline
\end{tabular}

Note-Values are presented as number (\%) unless indicated otherwise. 


\section{Table 2}

\section{Inclusion and Exclusion Criteria}

\section{Inclusion Criteria}

Subjects are status post amputation of an upper or lower limb (ie, amputation is not congenital)

Refractory PLP, despite conservative (nonsurgical) management for $>6$ months

Positive anesthetic/steroid block, as performed under CT guidance in an analogous fashion to planned cryoablation procedure

Ability and willingness of patient to provide written informed consent

PLP $=$ phantom limb pain

\section{Exclusion Criteria}

Active infection

Underlying congenital segmentation or other spinal anomalies that result in differential nerve root pressures

Significant spinal stenosis interpreted as "severe" on any cross-sectional imaging study

Pregnant or planning to become pregnant

Immunosuppression

History or laboratory results indicative of any significant cardiac, endocrine, hematologic, hepatic, immunologic, infectious, metabolic, urologic, pulmonary, gastrointestinal, dermatologic, psychiatric, renal, neoplastic, or other disorder that in the opinion of the principal investigator would preclude safe performance of cryoablation

Uncorrectable coagulopathies

Concurrent participation in another investigational trial involving systemic administration of agents or within the previous $30 \mathrm{~d}$

Previous surgical intervention-after amputation - that may have altered target nerve 


\section{Table 3}

Mean Pain Intensity Scores and Functional Status Ratings

\begin{tabular}{lccccc}
\hline & \multicolumn{2}{c}{ Pain Intensity } & & \multicolumn{2}{c}{ Functional Status } \\
\cline { 2 - 3 } \cline { 6 - 6 } Day & Mean & $(\mathbf{9 5 \%} \mathbf{C I})$ & & Mean & $\mathbf{( 9 5 \%} \mathbf{~ C I})$ \\
Baseline & 6.2 & $(5.2,7.3)$ & & 11.3 & $(8.8,13.9)$ \\
7 & 5.4 & $(4.2,6.6)$ & & 9.4 & $(7.3,11.5)$ \\
45 & 2.3 & $(1.6,3.0)$ & & 3.3 & $(2.2,4.4)$ \\
LT-FU & 2.0 & $(0.9,3.0)$ & & - & - \\
\hline
\end{tabular}

Note-Pain intensity was measured using the visual analog scale, and functional status was quantified using the Roland Morris Disability Questionnaire adapted for phantom limb pain. LT-FU assessment occurred at a mean of $194 \mathrm{~d} \pm 99$ (approximately 6.5 months) after the procedure. Functional status was not assessed at LT-FU assessment.

$\mathrm{CI}=$ confidence interval; $\mathrm{LT}-\mathrm{FU}=$ long-term follow-up. 


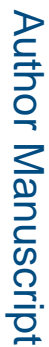
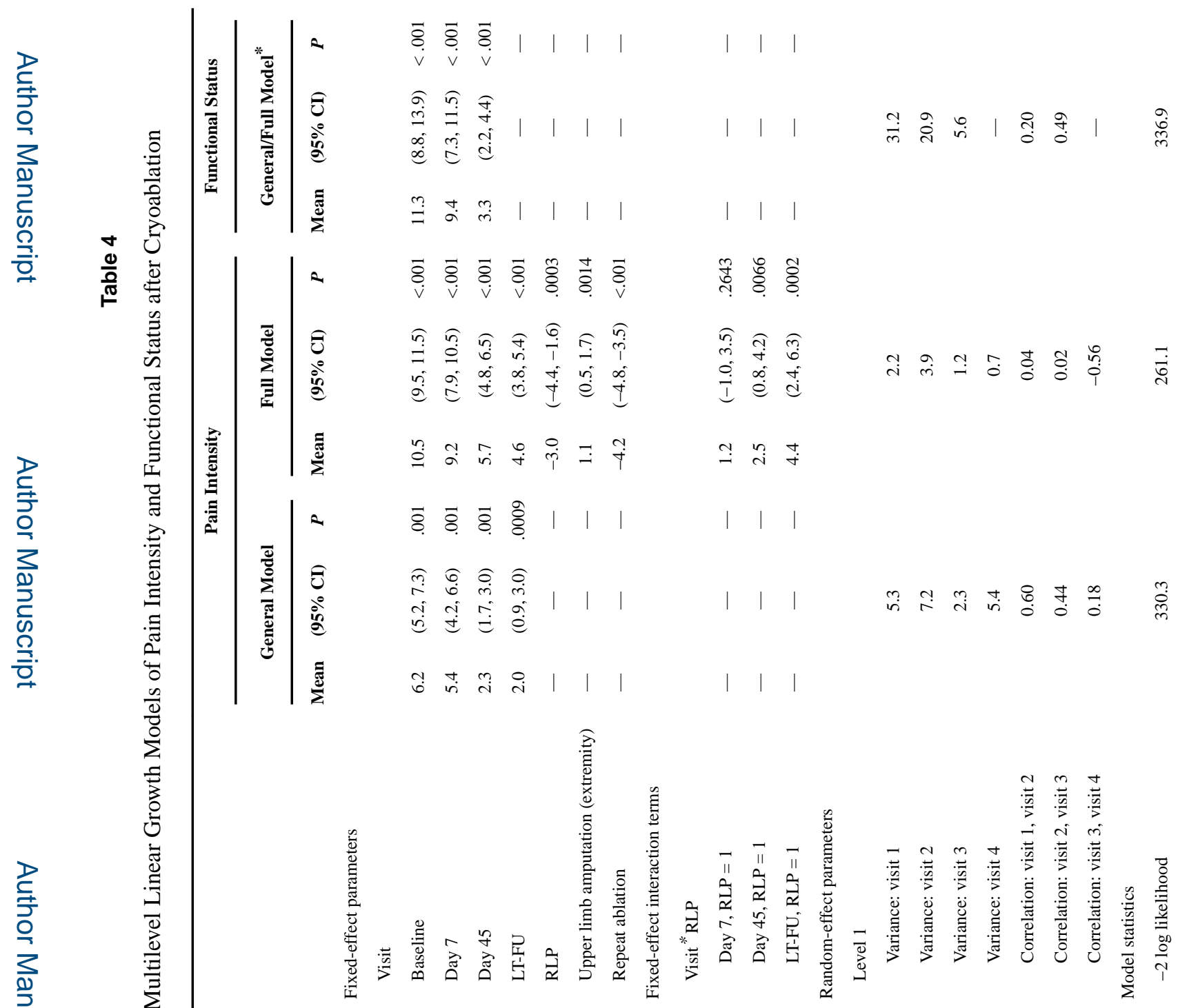

J Vasc Interv Radiol. Author manuscript; available in PMC 2017 December 28. 
Prologo et al.

Page 23

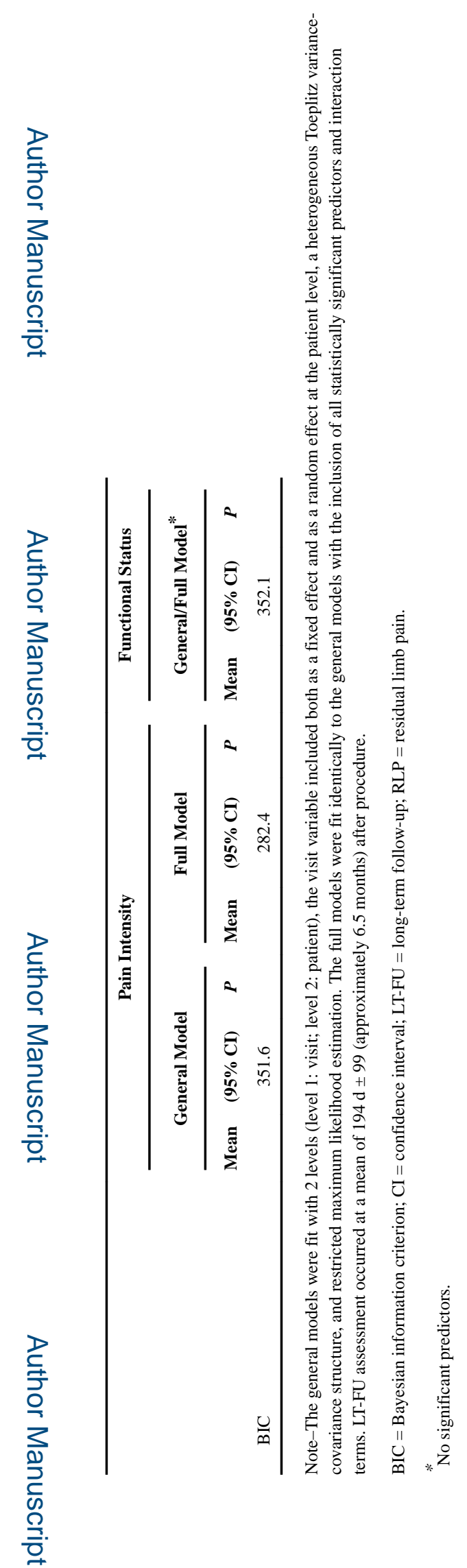

J Vasc Interv Radiol. Author manuscript; available in PMC 2017 December 28. 


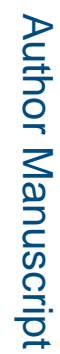

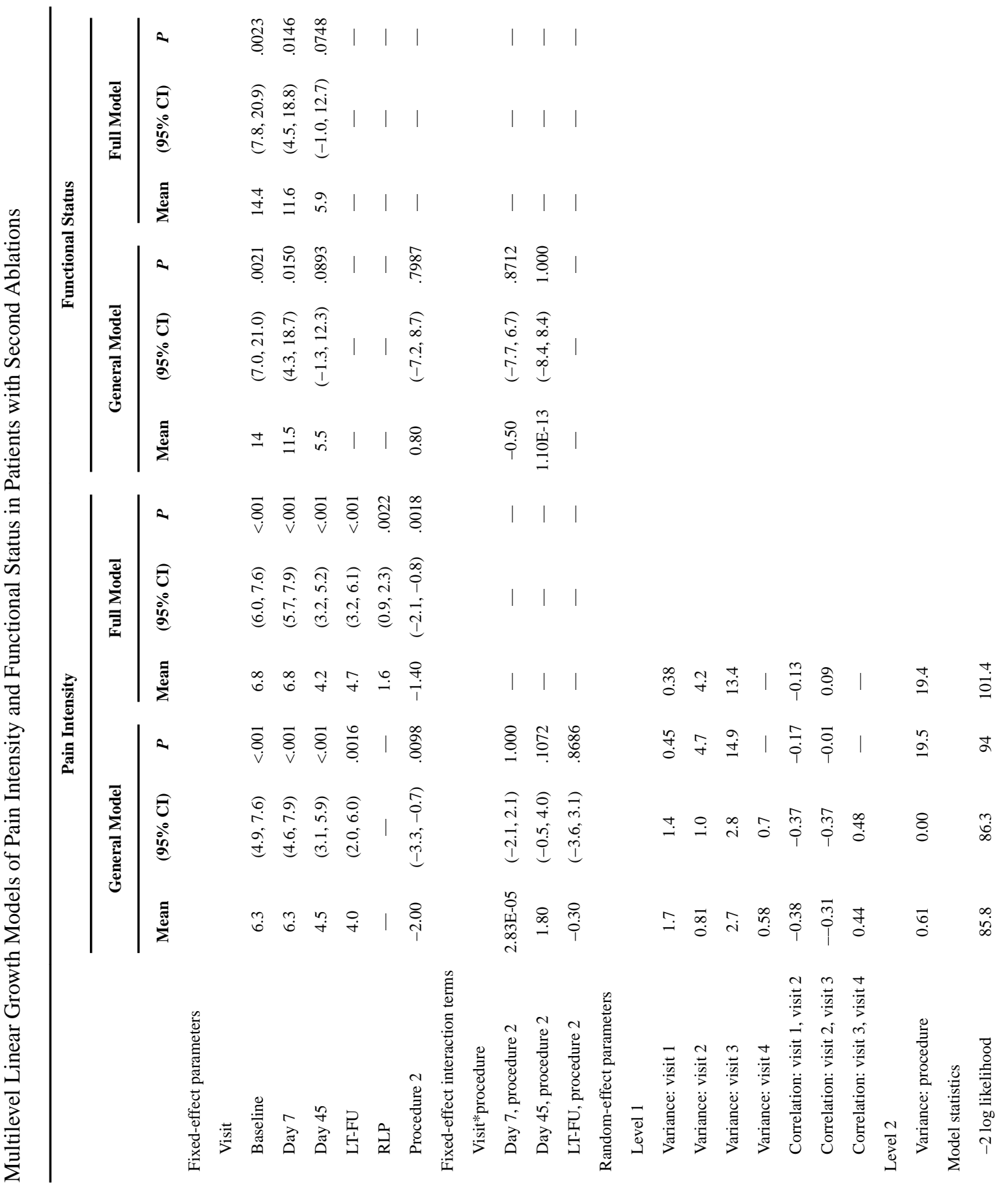


Prologo et al.

Page 25

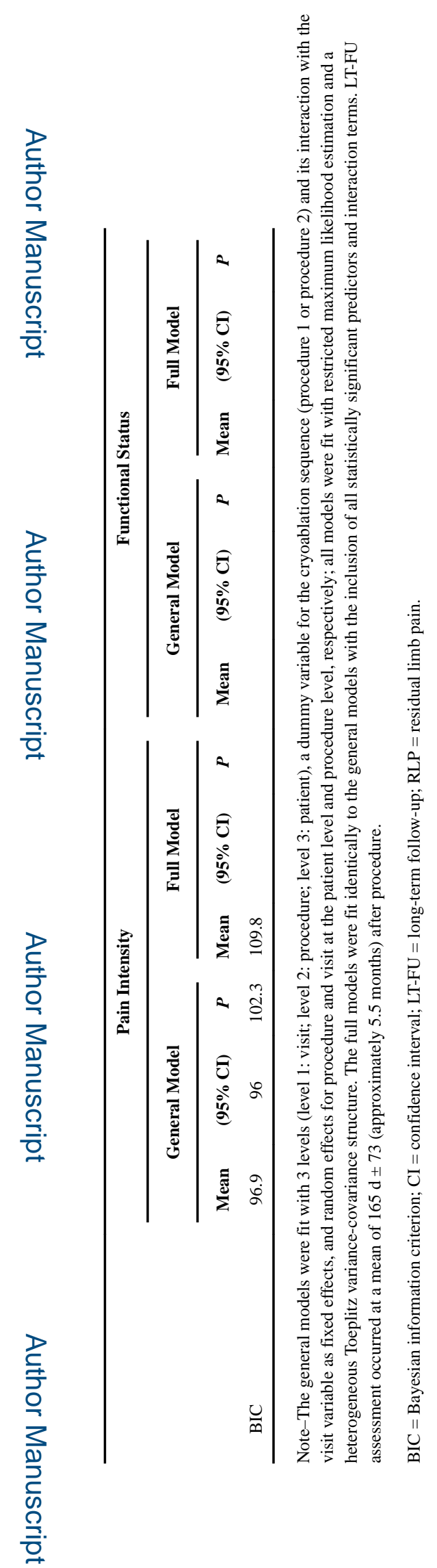

J Vasc Interv Radiol. Author manuscript; available in PMC 2017 December 28. 